\title{
Back to Basics in the Fight Against Tobacco
}

\author{
Heidi Rathjen, BEng, LLD, Drhc
}

Years of work by health organizations culminated with the passage of Bill C-71 on April 16th by the Senate of Canada. The legislation, championed by then Health Minister David Dingwall, severely restricts direct tobacco advertising, moderately restricts tobacco sponsorships and empowers the government to regulate tobacco in various other ways, including the control of nicotine levels in cigarettes.

However, the victory was short-lived in light of an immediate promise for further concessions for tobacco-funded events by the Prime Minister, through the regulatory vacuum that translates many sections of the law into de facto "voluntary codes", and in light of the instantaneous court challenge by the industry. Clearly, the battle for federal tobacco controls continues. The health community was virtually unanimous in perceiving the legislation as a substantial compromise, and much of its support rested on the potential rather than the actual health benefits of the Bill.

\section{David Dingwall and Allan Rock}

Notwithstanding the Bill's flaws, Mr. Dingwall is to be commended for his solid resilience in the face of the fierce, sometimes acerbic, opposition generated by the cigarette makers. In politics, as in the public debate, tobacco is less about health and more about public perception and political influence, both of which can be bought with enough money.

With good cause, high hopes now rest on the shoulders of the new Health Minister, Allan Rock. This former Justice Minister has already demonstrated integrity and resolve in his handling of gun con-

Campaign Director

Quebec Coalition for Tobacco Control Montreal trol. Although the gun lobby's resources and political savvy do not compare with those of the tobacco industry, Mr. Rock stood firm in the face of angry gun owners, occasionally vicious media, and many hostile colleagues, to deliver what health and safety experts lauded as complete and effective gun control legislation. The departure of Mr. Dingwall may represent the loss of a powerful ally for the health community, but there is hope that the appointment of Mr. Rock will bring renewed vigour to the federal fight against tobacco.

\section{Industry Sponsorship}

The strength of the tobacco industry is well known, but its show of force during the C-71 battle took many by surprise. Tobacco manufacturers managed to mobilize an impressive variety of groups and personalities to fight on their behalf. The Alliance for Sponsorship Freedom, in particular, dominated the public debates, in effect eclipsing the issue of the product and its victims. Not only did it convince most of the media, citizens and politicians that the Bill would bring about the cancellation of the country's most popular festivals and sporting events, but it managed to characterize tobacco opponents as single-minded "health Nazis" (in Quebec, "Ayatollahs de la santé") who were prepared to do anything to impose their views.

The Alliance is, of course, financed by the tobacco industry and organized by its public relations firm, Edelman Worldwide. Its spokespersons are reputable personalities who oppose the restrictions on tobacco promotion and, to the delight of tobacco executives, also vehemently deny the objectives and effectiveness of tobacco promotion through sponsorship. They joined advertising industry associations, retail stores and boards of trade in predicting massive job losses, curtailed freedom of expression and excessive government intrusion. Through their collaboration and the media's daily one-sided reports on their concerns, the industry successfully hijacked the debate about health issues, turning it into a debate about economics, morals and individual freedoms. Particularly in Quebec, the anti-smoking measures were portrayed as excessive state interference, a step on the slippery slope toward "banning coffee and butter".

\section{The Need to Legislate}

Yet basic common sense, largely disregarded in this debate, recognizes that in any democratic society the government must protect the population from major health risks - so much so that we take it for granted.

For instance, the federal Food and Drug Act ensures the safety of food and drugs, coffee and butter included. The Hazardous Products Act covers all other goods that entail a risk of some sort (such as toys, electronic equipment and hockey helmets). In Quebec, the Code national du bâtiment, the Règlement sur la qualité du milieu de travail and the Code de la securité routière establish the safety rules that permeate our daily environment.

Yet far from being the object of excessive regulation, tobacco is the one outstanding exception to all of these rules. If the legislation is to be consistent, then there is a definite need for much stronger measures than those provided by Bill C-71.

As the battle moves to the regulatory and implementation level, tobacco control proponents need to strengthen their efforts on two levels. 


\section{Back to Basics}

First, all public and political interventions must include a "back to the basics" component. Members of the health community must recognize that even if they are saturated with information on the enormous dangers of smoking, such awareness is obstructed in the general population by the omnipresence of tobacco advertisements and promotion, and by the social acceptance with which it is greeted in many regions (such as Quebec).

Amid the wide variety of non-healthcentred debates surrounding the legislation, it is essential that the basic relevant facts be firmly re-emphasized: tobacco kills over 40,000 Canadians every year; tobacco kills more people than alcohol, automobile accidents, AIDS, suicides, homicide and other drugs combined; tobacco is the number one preventable cause of death in our society; tobacco is the only legal product that causes addiction, disease and death when used exactly according to the manufacturer's intentions; there is no safe level of tobacco consumption; second-hand tobacco smoke can kill non-smokers; and finally, tobacco serves no useful purpose.

The tobacco industry has no effective arguments to counter these facts and always tries to avoid discussing them. Unfortunately, the lack of novelty also makes the arguments uninteresting to the media. Special efforts must therefore be made to insist that these facts be brought to light during every public debate and every encounter with politicians, many of whom are ill-informed on the severity of the issue.

\section{Fighting on the Industry's Turf}

On the second level, the tobacco industry is too powerful on its preferred battlefields for these to be ignored by the health community. Not only must we attack the credibility of the industry and its allies, we must also confront their economic, legal and personal freedom arguments. In fact, the health side comes out ahead on every single one of these fronts:

- On the economic front: Although the tobacco legislation is concerned with health and not financial matters, measures that reduce or prevent tobacco consumption nevertheless have an overall positive impact. According to the World Bank, "tobacco is, globally, a net economic disaster." 1 The health care costs from tobacco alone are much higher than the revenues generated by taxes on cigarettes. Contrary to popular belief, cigarette consumption actually reduces employment because of the high level of automation in the tobacco industry and the low "product versus taxes" value of cigarettes as compared with other products that smokers would spend their capital on and that yield higher economic activity (Pierre Fortin, economics professor: Université de Québec, Montréal, 1996).

- On the freedom of choice front: In spite of the industry's claim that informed adults should be allowed to smoke if they choose, the reality is that up to $90 \%$ of smokers start while in their teens. Moreover, the freedom to choose means very little to smokers addicted to nicotine who cannot quit.

- On the legal front: In Canada, freedom of expression is not absolute and can be subject to " reasonable limits " that can be justified in a free and democratic society. ${ }^{2}$ When the Supreme Court ruled that cigarette adverts could not be banned outright without sufficient justification, it nevertheless confirmed the government's duty to restrict tobacco advertising for public heath reasons.

- On the cultural front: The industry- funded public relations exercises have created the public perception that tobacco sponsorships bring in more revenues than they actually do. In reality, about $40 \%$ of the sponsored events get less than $1 \%$ of their revenues from cigarette manufacturers, and $50 \%$ get 1 to 9\%. ${ }^{3}$ Accordingly, the outright abolition of tobacco sponsorships will not be a death warrant for the majority of events.

All of these arguments mean that health advocates must enlist the participation of experts and groups in other sectors. Such support exists: in Quebec, which is the traditional stronghold of the Canadian tobacco industry, over 650 community organizations (including cultural groups) have endorsed the position of the Quebec Coalition for Tobacco Control. Their support and involvement will be crucial when the Quebec Health Minister, Dr. Jean Rochon, tables his much anticipated and possibly precedentsetting provincial legislation, most likely this fall.

In the meantime, despite the wellknown health hazards of smoking, young people are still picking up the habit, and addiction still ensures that they will keep at it for a long time. Tar and nicotine are not the main culprits of this epidemic, and the cure will not be found in medical laboratories or more public education. Tobacco is a social problem fueled by political reluctance to diminish the allure, availability, and addictive nature of cigarettes with gradual but unequivocal legislation.

\section{REFERENCES/BIBLIOGRAPHIE}

1. Barnum H. World Bank Policy on Tobacco. Washington, DC: World Bank, 1992.

2. Section 1, Canadian Charter of Rights and Freedoms. From the Constitution Act 1982.

3. Canadian Cancer Society. Canadian Conference of the Arts, March 1996 


\title{
Rappel des données de base dans la lutte contre le tabagisme
}

\author{
Heidi Rathjen, Beng, LLD, Drhc
}

Des années de travail par les organismes de la santé ont culminé par l'adoption au Sénat, le 16 avril dernier, du projet de loi C-71. Défendue par le ministre de la Santé de l'époque, David Dingwall, la nouvelle loi restreint sévèremment la publicité directe pour le tabac, limite dans une moindre mesure les commandites par l'industrie du tabac et habilite les gouvernements à réglementer le tabac d'autres façons, notamment en contrôlant la teneur en nicotine des cigarettes.

Toutefois, cette victoire a été de courte durée étant donné, d'une part, la promesse faite immédiatement par le Premier ministre d'accorder d'autres concessions aux activités financées par l'industrie du tabac, par le biais d'un vide juridique qui transforme de nombreux articles de la loi en "codes volontaires» de fait et, d'autre part, la contestation judiciaire intentée immédiatement par l'industrie du tabac. Il est clair que la bataille pour le contrôle du tabac par le gouvernement fédéral n'est pas encore gagnée et se poursuit. Pour pratiquement toute la communauté de la santé, cette loi représente déjà un très net compromis et la majeure partie du soutien qu'elle reçoit repose sur les bienfaits potentiels plus que réels de cette loi sur la santé.

\section{David Dingwall et Allan Rock}

Indépendamment des carences de la loi, monsieur Dingwall mérite d'être félicité pour l'opiniâtreté avec laquelle il a su résister à l'opposition acharnée, voire parfois acerbe, des fabriquants de cigarettes. En politique tout comme dans le débat public, le tabagisme est moins une question de santé que d'influence politique et de perception publique, deux choses qui s'achètent en y mettant les fonds nécessaires.

Non sans raison, de nombreux espoirs reposent maintenant sur le nouveau mi-

Directrice de campagne

Coalition québécoise pour le contrôle du tabac Montréal nistre de la Santé, Allan Rock. Cet ancien ministre de la Justice a déjà fait la preuve de son intégrité et de sa détermination dans sa façon de mener le dossier du contrôle des armes à feu. Bien que les ressources et la maitrise des arcanes de la politique dont dispose le lobby des armes à feu ne puissent se comparer à celles du lobby du tabac, monsieur Rock est resté ferme face à la colère des propriétaires d'armes à feu, face aux articles parfois malveillants des médias et face à l'hostilité de nombreux collègues, pour faire adopter ce que les experts de la santé et de la sécurité estimaient être une législation complète et efficace pour le contrôle des armes à feu. Le départ de monsieur Dingwall représente certes la perte d'un allié puissant de la communauté de la santé, mais on peut espérer que la nomination de monsieur Rock aura pour effet d'insuffler un nouvel élan à la lutte du gouvernement fédéral contre le tabac.

\section{Commandites par l'industrie}

La puissance de l'industrie du tabac n'échappe à personne, mais la démonstration de force qu'elle a faite durant la bataille pour l'adoption du projet de loi C71 en a surpris plus d'un. Les fabricants de tabac sont en mesure de mobiliser une gamme impressionnante de groupes et de personnalités pour défendre leur cause. Le Ralliement pour la liberté de commandite en particulier a dominé le débat public et a en fait réussi à éclipser la question du produit et de ses victimes. Non seulement, le Ralliement est-il parvenu à convaincre la majorité des médias, des citoyens et des dirigeants politiques que le projet de loi se traduirait par l'annulation de la plupart des festivals et événements sportifs les plus populaires du pays, mais il a aussi réussi à ce que les opposants au tabac soient vus comme des «nazis de la santé» (ou des «ayatollahs de la santé» au Québec), prêts à tout pour imposer leurs points de vue.
Bien entendu, le Ralliement est financé par l'industrie du tabac et géré par sa firme de relations publiques, Edelman Worldwide. Ses porte-parole sont des personnalités réputées qui s'opposent aux restrictions sur la promotion du tabac et qui, pour la plus grande joie des dirigeants de l'industrie du tabac, refusent avec véhémence de reconnaître l'efficacité et les objectifs réels de la promotion du tabac par le biais des commandites. Ils ont joint leurs voix à celles des associations de l'industrie publicitaire, des détaillants et des commissions du commerce pour prédire des pertes d'emplois massives, des restrictions à la liberté d'expression et un excès d'ingérence de l'État. Avec leur collaboration et celle de rapports tendancieux sur leurs inquiétudes, publiés presque quotidiennement dans les médias, l'industrie du tabac a réussi à détourner le débat sur les questions de santé et à l'orienter sur les questions économiques, la morale et les libertés individuelles. Au Québec tout particulièrement, les mesures anti-tabac ont été vues comme un excès d'ingérence de l'État, un premier pas sur une pente glissant vers «'interdiction du café et du beurre».

\section{Le besoin de légiférer}

Pourtant, il suffit d'un peu de bon sens, d'ailleurs largement oublié dans ce débat, pour reconnaître que dans toute société démocratique, le gouvernement a pour devoir de protéger la population contre ce qui menace sa santé - au point même que l'on tient cela pour acquis.

Ainsi, la Loi sur les aliments et drogues garantit l'innocuité des aliments et des médicaments, café et beurre compris. La Loi sur les produits dangereux s'applique à tous les autres produits qui présentent un risque quelconque (comme les jouets, l'équipement électronique et les casques des joueurs de hockey). Au Québec, le Code national du bâtiment, le Règlement sur la qualité du milieu de travail et le Code 
de la sécurité routière définissent les régles de sécurité qui régissent notre environnement quotidien.

Or, loin d'être l'objet d'un excès de règlementation, le tabac est la seule exception à toutes ces règles. Par conséquent, si nous voulons que la législation soit cohérente dans son ensemble, nous avons besoin de mesures beaucoup plus strictes que celles prévues par la Loi C-71.

$\mathrm{Au}$ moment où la bataille va se jouer au niveau des règlements et de l'application de la loi, les partisans du contrôle du tabac doivent redoubler d'efforts à ces deux niveaux.

\section{Rappel des données de base}

Pour commencer, toutes les interventions publiques et politiques doivent avoir un volet de «rappel des données de base». Il faut que les membres de la communauté de la santé se rendent compte que, s'ils sont pour leur part saturés d'informations sur les énormes dangers du tabagisme, tel n'est pas le cas pour le public en général car ces informations disparaissent derrière la promotion et les publicités omniprésentes sur le tabac, sans compter l'acceptation sociale dont bénéficie le tabac dans de nombreuses régions (comme le Québec).

Dans tous les débats suscités par cette législation et ne concernant pas spécifiquement la santé, il est essentiel d'insister de nouveau fermement sur les données de base : le tabac tue plus de 40000 Canadiens chaque année; le tabac tue davantage de personnes que l'alcool, les accidents de la route, le sida, les suicides, les homicides et la drogue ensemble; le tabac est la principale cause évitable de décès dans notre société; le tabac est le seul produit en vente libre dans notre société qui crée une accoutumance et entraîne la maladie et la mort lorsqu'il est consommé exactement comme le veut le fabriquant; il n'existe pas de niveau de consommation sans danger avec le tabac; la fumée de tabac ambiante peut tuer des non-fumeurs; enfin, le tabac n'a aucune utilité.

L'industrie du tabac ne dispose d'aucun argument pour contredire ces faits et cherche toujours à éviter d'en discuter. Les médias malheureusement n'accordent guère d'intérêt à ce qui n'est pas nouveau. Il faut donc déployer des efforts tout parti- culiers pour faire en sorte que cette réalité ressorte clairement dans chaque débat public et rencontre avec des politiciens, car un grand nombre d'entre eux ne mesurent pas la gravité du problème.

\section{Attaquer l'industrie sur son terrain}

Au second niveau, l'industrie du tabac est trop puissante sur son champ de bataille préféré pour que la communauté de la santé puisse se permettre de l'ignorer. Nous devons non seulement attaquer la crédibilité de l'industrie et de ses alliés, mais aussi remettre en cause leurs arguments économiques, juridiques et relatifs à la liberté de l'individu. En fait, sur chacun de ces fronts, c'est la communauté de la santé qui l'emporte:

- Sur le front économique : même si la législation sur le tabac concerne la santé et non les questions financières, les mesures prises pour réduire ou prévenir le tabagisme ont en fin de compte un résultat global positif. Selon la Banque mondiale, «globalement, le tabac constitue une ca-

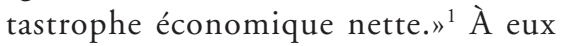
seuls, les coûts des soins de santé engendrés par la consommation de tabac sont beaucoup plus élevés que les revenus générés par les taxes sur les cigarettes. Contrairement à ce que l'on pense généralement, la consommation de cigarettes réduit le nombre d'emplois en raison du degré élevé d'automatisation de l'industrie du tabac d'une part et, d'autre part, du faible rapport "produit-taxes» des cigarettes, en comparaison avec d'autres produits que les fumeurs pourraient acheter et qui produisent davantage d'activités économiques (Pierre Fortin, professeur d'économie : Université de Québec, Montréal, 1996.)

- Sur le front de la liberté de choix : en dépit des affirmations de l'industrie comme quoi des adultes bien informés doivent avoir le droit de fumer s'ils le désirent, il n'en reste pas moins que $90 \%$ des fumeurs commencent à fumer lors de l'adolescence. En outre, la liberté de choix ne signifie pas grand chose pour les fumeurs accoutumés à la nicotine qui ne parviennent pas à s'arrêter.

- Sur le front juridique : au Canada, la liberté d'expression n'est pas absolue et peut être circonscrite à des «limites raisonnables» pouvant se justifier dans une société libre et démocratique. ${ }^{2}$ Lorsque la Cour suprême a décidé que les publicités sur les cigarettes ne pouvaient pas être formellement interdites faute de justifications suffisantes, elle a tout de même confirmé l'obligation qu'a le gouvernement de restreindre les publicités sur le tabac pour des raisons de santé publique.

- Sur le front culturel : les exercices de relations publiques financées par l'industrie ont réussi à donner l'impression au public que les commandites de l'industrie du tabac sont plus lucratives qu'elles ne le sont réellement. En réalité, près de $40 \%$ des activités commanditées reçoivent à peine $1 \%$ de leurs revenus des fabricants de cigarettes, et pour $50 \%$ d'entre elles, cela ne représente que de 1 à $9 \% .^{3}$ En conséquence, l'abolition totale des commandites par l'industrie du tabac ne sonnerait certainement pas le glas pour la majorité des événements.

Tous ces arguments signifient que les défenseurs de la santé doivent obtenir la participation d'autres experts et groupes dans les autres secteurs. Pareil soutien existe déjà : au Québec, le bastion traditionnel de l'industrie canadienne du tabac, plus de 650 organismes communautaires (dont des groupes culturels) ont donné leur appui à la Coalition québécoise pour le contrôle du tabac. Leur appui et leur participation vont être d'une importance cruciale lorsque le ministre de la Santé du Québec, le docteur Jean Rochon, déposera plus tard cet automne un projet de loi provincial qui suscite beaucoup d'anticipation et pourrait créer un précédent.

Dans l'intervalle, en dépit des risques bien connus du tabagisme pour la santé, les jeunes continuent de prendre l'habitude de fumer et leur accoutumance garantit que cette habitude se maintiendra pendant très longtemps. Le goudron et la nicotine ne sont pas les principaux coupables de cette épidémie, et le moyen de guérison ne viendra pas des laboratoires médicaux ou d'une plus grande sensibilisation du public. Le tabac est un problème social, alimenté par la mauvaise volonté du gouvernement à réduire l'attrait, la disponibilité et la nature accoutumante des cigarettes par l'application de mesures législatives graduelles mais sans équivoque. 